Said Gounane*, Yassir Barkouch, Abdelghafour Atlas, Mostafa Bendahmane, Fahd Karami and Driss Meskine

\title{
An adaptive social distancing SIR model for COVID-19 disease spreading and forecasting
}

https://doi.org/10.1515/em-2020-0044

Received October 19, 2020; accepted February 3, 2021; published online March 10, 2021

\begin{abstract}
Recently, various mathematical models have been proposed to model COVID-19 outbreak. These models are an effective tool to study the mechanisms of coronavirus spreading and to predict the future course of COVID-19 disease. They are also used to evaluate strategies to control this pandemic. Generally, SIR compartmental models are appropriate for understanding and predicting the dynamics of infectious diseases like COVID-19. The classical SIR model is initially introduced by Kermack and McKendrick (cf. (Anderson, R. M. 1991. "Discussion: the Kermack-McKendrick Epidemic Threshold Theorem." Bulletin of Mathematical Biology 53 (1): 3-32; Kermack, W. O., and A. G. McKendrick. 1927. "A Contribution to the Mathematical Theory of Epidemics." Proceedings of the Royal Society 115 (772): 700-21)) to describe the evolution of the susceptible, infected and recovered compartment. Focused on the impact of public policies designed to contain this pandemic, we develop a new nonlinear SIR epidemic problem modeling the spreading of coronavirus under the effect of a social distancing induced by the government measures to stop coronavirus spreading. To find the parameters adopted for each country (for e.g. Germany, Spain, Italy, France, Algeria and Morocco) we fit the proposed model with respect to the actual real data. We also evaluate the government measures in each country with respect to the evolution of the pandemic. Our numerical simulations can be used to provide an effective tool for predicting the spread of the disease.
\end{abstract}

Keywords: COVID-19 outbreak; forecasting; nonlinear SIR model; parameter estimations; social distancing.

\section{Introduction}

The new coronavirus (COVID-19 virus) that causes acute respiratory syndrome has spread to more than 180 countries. This virus has affected more than 13 million people and caused the death of more than 500.000 people until 14th July, and has also affected the world economy. The World Health Organization (WHO) named pneumonia caused by the new coronavirus COVID-19. The International Committee on Taxonomy of Viruses named it SARS-CoV-2 and it is similar to SARS-CoV and MERS-CoV. The virus SARS-CoV first occurred from November 2002 to June 2003 in Guangdong, China, and spread to many parts of the world. The virus MERS-CoV was found in 2012 in Saudi Arabia. Coronavirus can rise severe health problems and considerable economic consequences in various countries. In the fight against this coronavirus and the absence of a vaccine or treatment, the international community has more interest in putting in place tools that illustrate the principle

*Corresponding author: Said Gounane, Ecole Supéieure de Technologie d’Essaouira Km 9, Route d’Agadir, Essaouira Aljadida BP. 383, Essaouira, Morocco, E-mail: s.gounane@uca.ma

Yassir Barkouch, Fahd Karami, Driss Meskine, Ecole Supéieure de Technologie d’Essaouira Km 9, Route d’Agadir, Essaouira, Morocco, E-mail: dr.meskine@uca.ac.ma (Y. Barkouch), fa.karami@uca.ma (F. Karami), dr.meskine@uca.ac.ma (D. Meskine) Abdelghafour Atlas, Ecole Nationale des Sciences appliquéss, Université Cadi Ayyad, Marrakech, Morocco, E-mail: a.atlas@uca.ma

Mostafa Bendahmane, Institut de mathématique de Bordeaux (IMB) and INRIA-Carmen Bordeaux Sud-Ouest, Université de Bordeaux, Bordeaux, France, E-mail: mostafa.bendahmane@u-bordeaux.fr 
of its virality and consequently to control the spread of the virus and/or to mitigate it. To stop coronavirus spreading, various government measures like social distancing have been implemented in the world. On one hand, the social distancing measures have positive effects on preserving health, as they are used to avoid invidious track down infected by the COVID-19 virus (cf. (Bootsma and Ferguson 2007; Ferguson et al. 2020)). Another positive sides of social distancing are reduced traffic, fewer accidents, lower air pollution, resulting in reduced chances of respiratory diseases and possibly slowing down global warming. On the other hand, social distancing might result in a significant drop in physical activity which would increase levels of obesity, diabetes, cardiovascular diseases and a considerable economic consequences. Hence, assessment of the impact of social distancing remains an urgent challenge. This global health crisis of COVID-19 has brought to light the primordial role of the development of research and scientific evolution. In particular, the role of mathematical modeling to understanding the spread of the disease over time. In front of this situation of the spread of COVID-19 on an international scale, access is now facilitated to much more data and even to analyses carried out by scientists and researchers around the world. However, modeling and forecasting the spread of COVID-19 remains an urgent challenge to draw up data over the long term.

The SIR model is a famous mathematical model used in epidemiology to forecast the spread of a diseases. This model aims to compute the number of susceptible, infected and removed individuals on the basis of the probability of the disease spreading and the rates of transmission, recovery and recovered. They can also predict the maximum of infected and dead individuals only by virus per day. In the literature, one can be found various versions and mathematical studies for instance, in (Chou and Friedman 2016; Friedman 2018; Hethcote 2000; Murray 2002; WEISS 2009).

In this work, we propose a nonlinear adaptive system to model the spread of COVID-19 and investigate the impact of social distancing on the propagation of this pandemic. We developed a new fixed point algorithm to compute numerically a solution of the proposed model. To test the robustness of our approaches, we used data provided by international scientific reports from different countries in the world (Germany, Spain, Italy, France, Algeria and Morocco). Results show that our model is more accurate and robust for fitting the observed data compared to the classical SIR model. In addition, the proposed model can be used to evaluate the impact of social distancing for various countries. Finally we want to mention that our model is able to reproduce the observed trajectories of the COVID-19 pandemic in scenarios of rapid increase and by exponential decline afterwards in the presence of social distancing.

\section{The classical SIR and SIRD models}

\section{The SIR model}

The SIR model is one of the most epidemiological compartmental models used for the investigation of the spread of disease caused by a virus. This model was introduced by Kermack and McKendrick in 1927 to describe the evolution of the relative proportions of three disjoint classes which change with time $t$ (Figure 2.1).

In the SIR model, the population is subdivided into the following three epidemiologically distinct types of individuals and these classes are be represented by

1. The susceptible class $\mathbf{S}(t)$ the individuals have not yet been infected by the disease of interest.

2. The infected class $\mathbf{I}(t)$ the individuals who are currently infected and capable to transmit the disease to others.

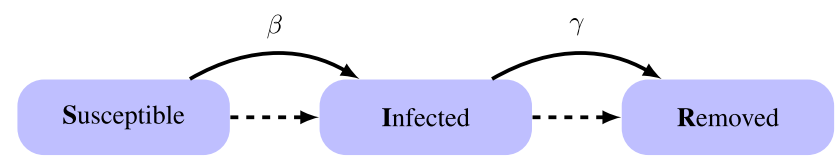

Figure 2.1: Compartimental diagram for SIR model. 
3. The removed class $\mathbf{R}(t)$ the individuals formally infected who are deceased, or have recovered and are permanently immune.

Many hypotheses are being assumed in the model, including a large and closed population, the outbreak is relatively short lived, we neglect the natural births-deaths, the absence of latent period and the healing after infection grant a lifetime immunity. It is assumed also that members of both susceptible and infectious populations are homogeneously distributed in space. In other words, every individual will be meeting every other individual per unit time with equal probability. Mathematically, the model can be formulated as a system of three equations (Kermack and McKendrick 1927):

$$
\left\{\begin{array}{l}
\frac{\mathrm{d} S(t)}{\mathrm{d} t}=-\frac{\beta}{N} S(t) I(t) \\
\frac{\mathrm{d} I(t)}{\mathrm{d} t}=\frac{\beta}{N} S(t) I(t)-\gamma I(t) \\
\frac{\mathrm{d} R(t)}{\mathrm{d} t}=\gamma I(t) .
\end{array}\right.
$$

Herein, the term $\frac{\beta}{N} S(t) I(t)$ represents the disease transmission rate by contact between susceptible and infected individuals. This means that the rate of come across between susceptible and infected individuals is proportional to the product of the population sizes. The parameter $\beta$ is the infection rate (to be estimated) and $\gamma$ is the recovery rate which can be computed with respect to the period of viral shedding. Adding all equations in the model, we observe that the total population $N=S+I+R$ is a constant. Indeed, we see that

$$
\frac{\mathrm{d} S(t)}{\mathrm{d} t}+\frac{\mathrm{d} I(t)}{\mathrm{d} t}+\frac{\mathrm{d} R(t)}{\mathrm{d} t}=0 \quad \text { implies } S(t)+I(t)+R(t)=N \text { for each time } t .
$$

The system (2.1) are completed by the initial conditions representing the first infected individual appeared in the population, which correspond to $S\left(t_{0}\right)=N, I\left(t_{0}\right)=0$ and $R\left(t_{0}\right)=0$. Note that, the case where the number of infected and recovered are neglected with respect to the total population the number of susceptible $S$, we obtain the following approximation: $S(t) \sim S\left(t_{0}\right)=N$. Observe that, the first Eq. (2.1) can be written as

$$
\frac{\mathrm{d} I(t)}{\mathrm{d} t} \sim \beta I(t)-\gamma I(t)
$$

Therefore, we obtain an ordinary differential equation with constant coefficients and the solution can be expressed as

$$
I(t) \sim I\left(t_{0}\right) \exp ((\beta-\gamma) t)
$$

The doubling time, which represents the number of infected people can be doubled over each time interval of length $T_{d}=\ln (2) /(\beta-\gamma)$. Based on available data, this exponential growth is observed in the first days of starting COVID-19 outbreak data from various countries, see the following figure (Figure 2.2):

For more analysis about Classical SIR model, see for instance (Hethcote 2000; Murray 2002; WEISS 2009) and the references therein and the model has been proposed recently to forecasting COVID-19 pandemic in (Bertozzi et al. 2020).

\section{The SIRD model}

Now regarding system (2.1), we can extend this SIR model including an additional class $D(t)$ of deceased individuals due to the pandemic and $\sigma$ the mortality rate of the infected (Figure 2.3).

The dynamic of SIRD model is governed by the following system of ODEs

$$
\left\{\begin{array}{l}
\frac{\mathrm{d} S(t)}{\mathrm{d} t}=-\frac{\beta}{N} S(t) I(t) \\
\frac{\mathrm{d} I(t)}{\mathrm{d} t}=\frac{\beta}{N} S(t) I(t)-(\gamma+\sigma) I(t) \\
\frac{\mathrm{d} R(t)}{\mathrm{d} t}=\gamma I(t), \quad D(t)=N-S(t)-I(t)-R(t) .
\end{array}\right.
$$



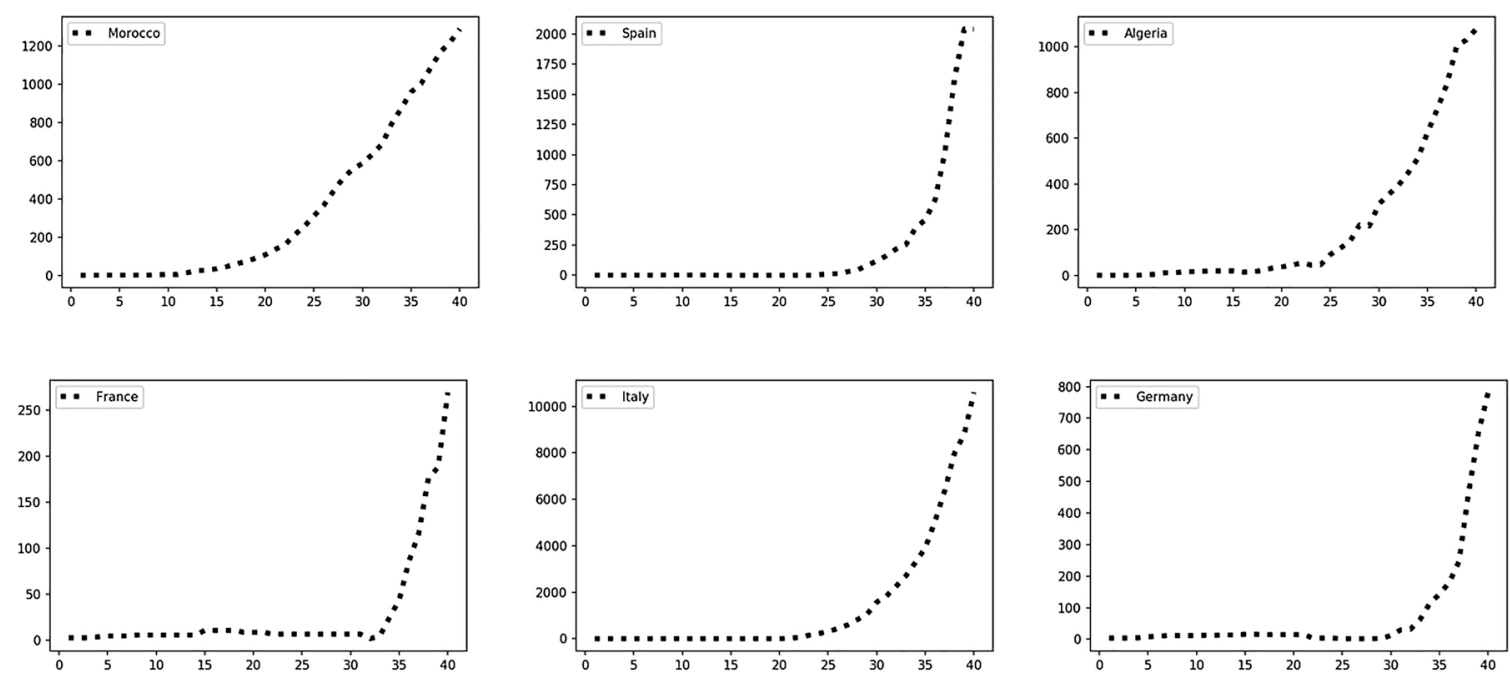

Figure 2.2: Reported infective data during first 40 days since the appears of the first case.

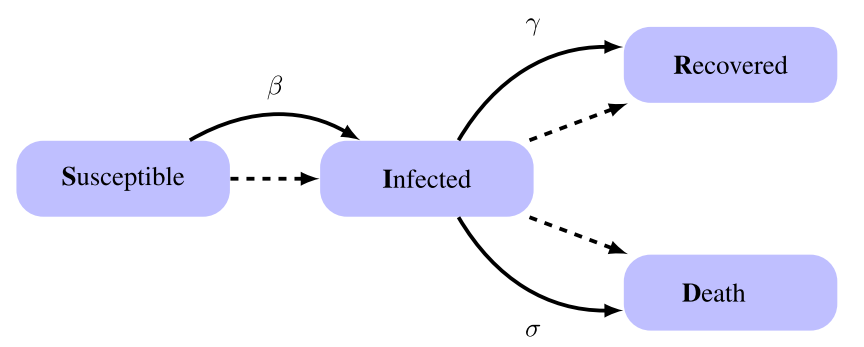

Figure 2.3: Compartmental diagram for SIRD model.

We observe that the total population $S(t)+I(t)+R(t)+D(t)=N$ is a constant for each time $t$. Now, thanks to (Murray 2002), the basic reproduction number of (2.4) can be computed by

$$
\mathcal{R}_{0}=\frac{\beta}{\gamma+\sigma} .
$$

Similarly to subsection (2.1), we arrive to $I(t) \sim I\left(t_{0}\right) \exp ((\beta-\gamma-\sigma) t)$ for $S\left(t_{0}\right) \sim N$. In this case, it is easy to see that the number of infected population will grow for $\beta>\gamma+\sigma$ (i.e. $\mathcal{R}_{0}>1$ ) and when $\beta<\gamma+\sigma$ (i.e. $\mathcal{R}_{0}<1$ ) it will decay which plays the key role behind disease propagation. On the other hand, thanks to (2.4), the cumulative infections can be computed by $I_{c}(t)=I(t)+R(t)+D(t)$ and the evolution equation of $I_{c}$ can be written as

$$
\frac{\mathrm{d} I_{c}(t)}{\mathrm{d} t}=\frac{\beta}{N} S(t) I(t)
$$

Integrating this equation, we observe that $I_{c}(t) \sim \frac{\beta}{\beta-\gamma-\sigma} I\left(t_{0}\right) \exp ((\beta-\gamma-\sigma) t)$ for $S(t) \sim N$.

\section{The proposed non-linear SIRD model}

\section{The non-linear SIRD model}

In order to reduce the impact of COVID-19, government measures intended to modify human behavior, such as the social distancing strategy that can help to slow the spread of COVID-19. Various restrictive measures 
adopted for the coronavirus infection suggest to introduce some new models of the infection spreading. Inspired by (Eksina, Paarpornb, and Weitz 2019), our goal in this work is to introduce a new model taking account of human behavior to the propagation of diseases and which individuals reduce their contacts with the infected individuals. Now, we propose the following modified SIRD model where the social distancing is taken into account

$$
\left\{\begin{array}{l}
\frac{\mathrm{d} S(t)}{\mathrm{d} t}=-\beta \mathcal{L}\left(t, \frac{S(t)}{N}\right) I(t), \\
\frac{\mathrm{d} I(t)}{\mathrm{d} t}=\beta \mathcal{L}\left(t, \frac{S(t)}{N}\right) I(t)-(\gamma+\sigma) I(t), \\
\frac{\mathrm{d} R(t)}{\mathrm{d} t}=\gamma I(t), \quad D(t)=N-S(t)-I(t)-R(t) .
\end{array}\right.
$$

Herein, $\mathcal{L}$ is a non-linear continuous function that describe the social distancing and the sensitivity to the disease. This function is given in (3.2) below. Note that, in the case where the function $\mathcal{L}(t, r) \sim r$ for any $r, t \in \mathbb{R}^{+}$, we get the SIRD model (2.4). We would like to mention that after the pandemic starts to propagate, there is a large reduction in the population $S(t)$ by social distancing measures. For that, we assume that $\mathcal{L}(t, r) \sim r$ for $t \leq t_{c}$ and $\mathcal{L}(t, r) \sim r h(r)$ for $t \geq t_{c}$, where $t_{c}$ is the time of beginning lockdown and $h(r)$ is a non decreasing function. A typical examples of the function $\mathcal{L}$ are

$$
\mathcal{L}(t, r)=r^{r_{\varepsilon}^{\delta}(t)} \quad \text { or } \quad \mathcal{L}(t, r)=r^{1+\varepsilon} \exp \left(r q^{\delta}(t)\right),
$$

where $p_{\varepsilon}^{\delta}(\cdot)>1$ and $q^{\delta}(\cdot) \geq 0$ are continuous functions such that

$$
p_{\varepsilon}^{\delta}(t)= \begin{cases}1+\varepsilon & \text { if } t \leq t_{c} \\ \frac{p^{\star}-1-\varepsilon}{\delta}\left(t-t_{c}\right)+1+\varepsilon & \text { if } t_{c} \leq t \leq t_{c}+\delta \\ p^{\star} & \text { if } t \geq t_{c}+\delta\end{cases}
$$

and

$$
q^{\delta}(t)= \begin{cases}0 & \text { if } t \leq t_{c}, \\ \frac{q^{\star}}{\delta}\left(t-t_{c}\right) & \text { if } \quad t_{c} \leq t \leq t_{c}+\delta, \\ q^{\star} & \text { if } t \geq t_{c}+\delta .\end{cases}
$$

Herein, $\varepsilon \sim 0$ is a regularized parameter and $\delta$ represents a period for which the lockdown is taking effect. Regarding the social distancing function $p_{\varepsilon}^{\delta}(t)$ (res. $q^{\delta}(t)$ ), it is clear that when the social distancing starts the proportion of susceptible individuals is reduced. Note that, before of the beginning of the outbreak at time $t_{c}$ (the time at which social distancing is started), we get easily from (3.1) $-(3.4) p_{\varepsilon}^{\delta}(t) \sim 1$ (res. $q^{\delta}(t) \sim 0$ ) and we deduce the classical SIRD model (2.4). From time $t_{c}$ to $t_{c}+\delta$, is the interval when social distancing measures are started. After time $t_{c}+\delta$, the social distancing measures are completely effective. Without loss of generality, we use the expression of $\mathcal{L}$ in the rest of this paper.

\section{The reproduction number and phase plane}

It is clear that our model has a non-trivial equilibrium of the system (3.1), given by $\left(S^{\star}, 0, R^{\star}, D^{\star}\right)$ where $S^{\star}+R^{\star}+D^{\star}=N$. Moreover, the pandemic will occur if the infected proportion increases, namely

$$
\mathcal{L}\left(t_{0}, \frac{S\left(t_{0}\right)}{N}\right)>\frac{\gamma+\sigma}{\beta} \text { i.e. } S\left(t_{0}\right)>N \mathcal{L}^{-1}\left(t_{0}, \frac{\gamma+\sigma}{\beta}\right)
$$

Recall that, the total population $S(t)+I(t)+R(t)+D(t)=N$ for each time $t \geq 0$. In the next step we focus only on the equations in $S$ and $I$. Following the works in (Driessche and Watmough 2002), the right side of the system (3.1) can be decomposed as $\mathcal{F}+\mathcal{V}^{+}+\mathcal{V}^{-}$where 


$$
\mathcal{F}(S, I)=\left(0, \beta \mathcal{L}\left(t, \frac{S(t)}{N}\right) I\right), \quad \mathcal{V}^{+}(S, I)=(0,0) \quad \text { and } \quad \mathcal{V}^{-}(S, I)=\left(-\beta \mathcal{L}\left(t, \frac{S(t)}{N}\right) I,-(\gamma+\sigma) I\right)
$$

Now, we let $F=\beta \mathcal{L}\left(t_{0}, \frac{S\left(t_{0}\right)}{N}\right)$ and $V=-(\gamma+\sigma)$ to deduce that

$$
\mathcal{R}_{0}=\rho\left(-F V^{-1}\right)=\frac{\beta}{\gamma+\sigma} \mathcal{L}\left(t_{0}, \frac{S\left(t_{0}\right)}{N}\right)=\frac{\beta}{\gamma+\sigma}
$$

where $\rho$ is a spectral radius. Moreover, the number of susceptible individuals that can get infected by an individual in the population at time $t$, can be represented by the following effective reproduction number

$$
\mathcal{R}_{t}=\frac{\beta}{\gamma+\sigma} \mathcal{L}\left(t, \frac{S(t)}{N}\right)
$$

Next, we study the phase plane of our model. We divide the first equation by the second in (3.1), to formulate the phase portrait to our system in the following equation

$$
\frac{\mathrm{d} I}{\mathrm{~d} S}=\frac{\gamma+\sigma}{\beta \mathcal{L}\left(t, \frac{S(t)}{N}\right)}-1:=\frac{1}{\mathcal{R}_{0} \mathcal{L}\left(t, \frac{S(t)}{N}\right)}-1
$$

For $\mathcal{L}\left(t, \frac{S}{N}\right)=\left[\frac{S}{N}\right]^{p_{\varepsilon}^{\delta}(t)}$, we integrate Eq. (3.9) with respect to $S$, to obtain

$$
\begin{aligned}
I(t) & =I\left(t_{0}\right)+\frac{1}{\mathcal{R}_{0}} \int_{t_{0}}^{t}\left[\frac{S}{N}\right]^{-p_{\varepsilon}^{\delta}(t)} \mathrm{d} S-\int_{t_{0}}^{t} \mathrm{~d} S \\
& =-S(t)+I\left(t_{0}\right)+S\left(t_{0}\right)+\frac{N}{\varepsilon \mathcal{R}_{0}}\left[\frac{N}{S\left(t_{0}\right)}\right]^{\varepsilon}+\frac{N}{\mathcal{R}_{0}\left(1-p_{\varepsilon}^{\delta}(t)\right)}\left[\frac{S(t)}{N}\right]^{1-p_{\varepsilon}^{\delta}(t)}
\end{aligned}
$$

for any time $t>t_{c}$. Notice that

$$
\frac{\mathrm{d}^{2} I}{\mathrm{~d} S^{2}}=\frac{-p_{\varepsilon}^{\delta}(t)}{N \mathcal{R}_{0}}\left[\frac{S}{N}\right]^{-p_{\varepsilon}^{\delta}(t)-1} \leq 0
$$

Therefore, the maximum $I_{\max }$ reach after $t_{c}+\delta$ (recall that $I_{\max }$ is obtained when $\frac{\mathrm{d} I}{\mathrm{~d} t}=0$ ) for $S^{\star}=N \mathcal{R}_{0}^{\frac{1}{p^{\star}}}$. Consequently, the maximum number of ineffective population is given by

$$
I_{\max }=-N \mathcal{R}_{0}^{\frac{1}{p^{\star}}}+I\left(t_{0}\right)+S\left(t_{0}\right)+\frac{N}{\varepsilon \mathcal{R}_{0}}\left[\frac{N}{S\left(t_{0}\right)}\right]^{\varepsilon}+\frac{N}{\left(1-p^{*}\right)}\left[\mathcal{R}_{0}\right]^{\frac{1}{p^{*}}-2}
$$

Moreover, the first infected individual appears in the population corresponding to $S\left(t_{0}\right)+I\left(t_{0}\right)=N$ and it follows

$$
I_{\max }=\frac{N}{\left(1-p^{*}\right)}\left[\mathcal{R}_{0}\right]^{\frac{1}{p^{*}}-2}+N\left(1+\frac{1}{\varepsilon \mathcal{R}_{0}}-\mathcal{R}_{0}^{\frac{1}{p^{\star}}}\right) .
$$

Now, dividing the first equation by the last one of (3.1), the phase portrait for the pandemic system, can be formulated as

$$
\frac{\mathrm{d} S}{\mathrm{~d}(R+D)}=-\frac{\beta}{\gamma+\sigma} \mathcal{L}\left(t, \frac{S(t)}{N}\right)=-\mathcal{R}_{0} \mathcal{L}\left(t, \frac{S(t)}{N}\right) .
$$

Now, integrating this equation with respect to $S$, we get

$$
R(t)+D(t)=R\left(t_{0}\right)+D\left(t_{0}\right)+\frac{N}{\varepsilon \mathcal{R}_{0}}\left[\frac{N}{S\left(t_{0}\right)}\right]^{\varepsilon}+\frac{N}{\mathcal{R}_{0}\left(1-p_{\varepsilon}^{\delta}(t)\right)}\left[\frac{S(t)}{N}\right]^{1-p_{\varepsilon}^{\delta}(t)}
$$

Letting $t \rightarrow \infty$, assuming $R\left(t_{0}\right)=D\left(t_{0}\right)=0$ and $S\left(t_{0}\right)=N$ and thanks to the fact that $I(\infty)=0$, we obtain $R(\infty)+D(\infty)=N-S(\infty)$. Additionally, the proportion of susceptible $S(\infty)$ can be computed by solving the following non-linear equation 


$$
\frac{S(\infty)}{N}+\frac{1}{\mathcal{R}_{0}\left(1-p^{\star}\right)}\left[\frac{S(\infty)}{N}\right]^{1-p^{\star}}=1-\frac{1}{\varepsilon \mathcal{R}_{0}} .
$$

\section{Numerical approches}

Generally, explicit treatment of (2.1) means stricter conditions on time step and the parameters to achieve stability of time discretization schemes. To overcome this, we will use the implicit Euler method, discretizing the time variable as $t=k \tau$ where $k$ is a positive number and $\tau$ is the time step. The discrete iterative scheme of the proposed model can be written as

$$
\left\{\begin{array}{l}
S_{k+1}=S_{k}-\tau \beta \mathcal{L}\left(t_{k+1}, \frac{S_{k+1}}{N}\right) I_{k+1}, \\
I_{k+1}=I_{k}+\tau \beta \mathcal{L}\left(t_{k+1}, \frac{S_{k+1}}{N}\right) I_{k+1}-\tau(\gamma+\sigma) I_{k+1}, \\
R_{k+1}=R_{k}+\tau \gamma I_{k+1} \\
D_{k+1}=N-S_{k+1}-I_{k+1}-R_{k+1},
\end{array}\right.
$$

where $S_{k+1}, I_{k+1}, R_{k+1}$ and $D_{k+1}$ are numbers of the susceptible, the infected and the recovered and death individuals in the specific day $k+1$ respectively. First, assume that $\mathcal{L}\left(t_{k+1}, \frac{S_{k+1}}{N}\right)=\frac{S_{k+1}}{N}$ for any $k \geq 0$, the discrete system (3.11) implies

$$
\left\{\begin{array}{l}
S_{k+1}=S_{k}-\tau \frac{\beta}{N} S_{k+1} I_{k+1} \\
I_{k+1}=I_{k}+\tau \frac{\beta}{N} S_{k+1} I_{k+1}-\tau(\gamma+\sigma) I_{k+1}
\end{array}\right.
$$

Now, we add the first and the second equations in (3.12) to obtain

$$
(1+\tau(\gamma+\sigma)) I_{k+1}=I_{k}+S_{k}-S_{k+1} .
$$

Using this and the first equation in (3.12), we deduce

$$
S_{k+1}=\frac{S_{k}}{1+\tau \frac{\beta}{N} I_{k+1}} .
$$

Replacing (3.14) in (3.13) and using the fact that $S_{k}+I_{k}+R_{k}+D_{k}=N$, we get

$$
I_{k+1}=\frac{\sqrt{\left(\tau(\gamma+\sigma)+1-\tau \frac{\beta}{N}\left(S_{k}+I_{k}\right)\right)^{2}+4 \tau \frac{\beta}{N}(\tau(\gamma+\sigma)+1) I_{k}}-\left(\tau(\gamma+\sigma)+1-\tau \frac{\beta}{N}\left(S_{k}+I_{k}\right)\right)}{2 \tau \frac{\beta}{N}(1+\tau(\gamma+\sigma))}:=\eta_{k} .
$$

Consequently, we have

$$
S_{k+1}=\frac{S_{k}}{1+\tau \frac{\beta}{N} \eta_{k}}, \quad R_{k+1}=R_{k}+\tau \gamma \eta_{k} \quad \text { and } \quad D_{k+1}=D_{k}+\tau \sigma I_{k+1}
$$

which can be computed directly. Now we take $\Theta_{k+1}:=\frac{N}{S_{k+1}} \mathcal{L}\left(t_{k+1}, \frac{S_{k+1}}{N}\right)$ to obtain from (3.11)

$$
\left\{\begin{array}{l}
S_{k+1}=S_{k}-\tau \frac{\beta}{N} \Theta_{k+1} S_{k+1} I_{k+1} \\
I_{k+1}=I_{k}+\tau \frac{\beta}{N} \Theta_{k+1} S_{k+1} I_{k+1}-\tau(\gamma+\sigma) I_{k+1}
\end{array}\right.
$$


Therefore, the solution of (3.17) can be obtained by using the following fixed point method

$$
\left\{\begin{array}{l}
S_{k+1}^{n+1}=S_{k}-\tau \frac{\beta}{N} \Theta_{k+1}^{n} S_{k+1}^{n+1} I_{k+1}^{n+1} \\
I_{k+1}^{n+1}=I_{k}+\tau \frac{\beta}{N} \Theta_{k+1}^{n} S_{k+1}^{n+1} I_{k+1}^{n+1}-\tau(\gamma+\sigma) I_{k+1}^{n+1} \\
R_{k+1}^{n+1}=R_{k}+\tau \gamma I_{k+1}^{n+1} \\
D_{k+1}=N-S_{k+1}-I_{k+1}-R_{k+1}
\end{array}\right.
$$

where $\Theta_{k+1}^{n}:=\frac{N}{S_{k+1}^{n}} \mathcal{L}\left(t_{k+1}, \frac{S_{k+1}^{n}}{N}\right)$ for $k, n \in \mathbb{N}$. We shall see further that all results obtained for scheme (3.13)-(3.15) are also true, with convenient adaptations, for the fully implicit scheme. Now, we present the algorithm used to solve our proposed model:

Algorithm 1: Fixed point iterative Algorithm for non linear proposed SIRD model.

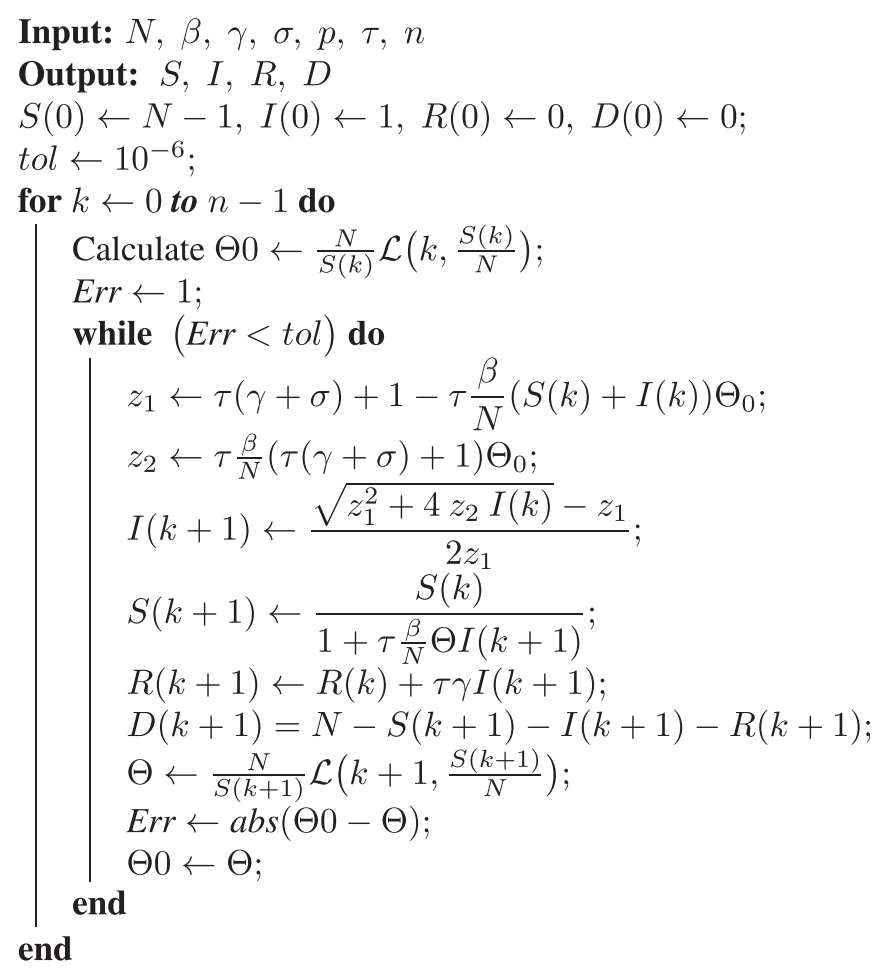

\section{Numerical simulations and results}

The purpose of this section is to exemplify and evaluate the model presented in the previous section. The implementation and the numerical solutions are realized by using python language. The datasets used in this paper are available for public (we use the data from WHO (http://www.who.int) and Moroccan Ministry of Health). The dataset contains the number of new and cumulative cases, deaths and recovered cases, together with the number of active cases of COVID-19 since January 20, 2020. We conducted simulations for various countries: Germany, Spain, Italy, France, Algeria and Morocco using the total population numbers $N$ as shown in Table 1. 
Table 1: Total population from WHO for various countries.

\begin{tabular}{rrrrrrr}
\hline & Germany & Spain & Italy & France & Morocco & Algeria \\
\hline $\mathrm{N}$ & 83.780 .597 & 46.754 .577 & 60.462 .730 & 65.271 .779 & 36.903 .169 & 43.837 .260 \\
\hline
\end{tabular}

\section{Parameter estimation}

In this subsection, we present the technic to fit the proposed model to COVID-19 data. We estimate the transmission rate $\beta$, the recovery rate $\gamma$, the death rate $\sigma$ and the distancing parameter $p^{\star}$. The model parameters $\vartheta:=\left(\beta, \gamma, \sigma, p^{\star}\right)$ can be estimated via least-square fitting of the model solution to the observed data (Liu and Stechlinski 2017). This can be expressed by finding for the set of parameters $\vartheta^{\star}:=\left(\beta^{\star}, \gamma^{\star}, \sigma^{\star}, p_{\star}^{\star}\right)$ that minimizes the sum of squared differences between the real data $\left(\zeta_{1}, \zeta_{2}, \ldots, \zeta_{k}\right)$ and the corresponding model solution named by $\left(\zeta_{\vartheta}\left(t_{1}\right), \zeta_{\vartheta}\left(t_{2}\right), \ldots, \zeta_{\vartheta}\left(t_{k}\right)\right)$. Consequently, the objective function is written as

$$
\begin{aligned}
\min _{\vartheta} \sum_{j=1}^{k}\left\|\zeta_{\vartheta}\left(t_{j}\right)-\zeta_{j}\right\|^{2}:= & \sum_{j=1}^{k} w_{1, t_{j}}\left(I_{C, \vartheta}\left(t_{j}\right)-I_{C, j}\right)^{2}+\sum_{j=1}^{k} w_{2, t_{j}}\left(I_{\vartheta}\left(t_{j}\right)-I_{j}\right)^{2} \\
& +\sum_{j=1}^{k} w_{3, t_{j}}\left(R_{\vartheta}\left(t_{j}\right)-R_{j}\right)^{2}+\sum_{j=1}^{k} w_{4, t_{j}}\left(D_{\vartheta}\left(t_{j}\right)-D_{j}\right)^{2},
\end{aligned}
$$

where $\zeta_{\vartheta}(t):=\left(I_{C, \vartheta}(t), I_{\vartheta}(t), R_{\vartheta}(t), D_{\vartheta}(t)\right)$. Herein, $\left(I_{\vartheta}(t), R_{\vartheta}(t), D_{\vartheta}(t)\right)$ is given by the solution of the system (2.1) and the cumulative infection is computed by $I_{C, \vartheta}(t)=I_{\vartheta}(t)+R_{\vartheta}(t)+D_{\vartheta}(t)$. The time of observed data is denoted by $t_{i}$, where $i$ is the number of data points. We accord more weight to smaller data points and the corresponding weight can expressed as $w_{1, t_{j}}=\frac{1}{I_{C, j}}, w_{2, t_{j}}=\frac{1}{I_{j}}, w_{3, t_{j}}=\frac{1}{R_{j}}, w_{4, t_{j}}=\frac{1}{D_{j}}$ for $j=1, \ldots, k-1$ and some alternative choices of the weight can be found in (Chowell 2017).

\section{Numerical tests}

Various countries has implemented social distancing measures to reduce the transmission of the outbreak between individuals. These types of measures have an impact on the susceptible numbers on the proposed

Table 2: The fitting parameter's of classical SIRD and our model.

\begin{tabular}{lrrrrrr}
\hline \multirow{2}{*}{ Quantity } & \multicolumn{9}{c}{} & & Country \\
\cline { 2 - 7 } & Germany & Spain & Italy & France & Morocco & Algeria \\
\hline$\beta_{p^{\star}}\left(\right.$ ind $^{-1}$ day $\left.^{-1}\right)$ & $0.23,731,968$ & $0.23,973,950$ & $0.22,182,590$ & $0.17,738,282$ & $0.21,154,361$ & $0.18,720,912$ \\
$\gamma_{p^{\star}}\left(\right.$ day $\left.^{-1}\right)$ & $0.05,906,339$ & $0.03,478,090$ & $0.01,856,929$ & $0.02,036,310$ & $0.04,175,114$ & $0.04,548,430$ \\
$\sigma_{p^{\star}}\left(\right.$ day $\left.^{-1}\right)$ & $0.00,295,122$ & $0.00,818,803$ & $0.00,755,085$ & $0.01,065,986$ & $0.00,137,191$ & $0.00,643,650$ \\
$p^{\star}$ & $887.661,008$ & $452.286,058$ & $710.098,741$ & $617.774,090$ & $9,821.747,734$ & $8,086.18,695$ \\
$\beta_{1}$ (ind $^{-1}$ day $\left.^{-1}\right)$ & $1.25,477,482$ & $1.49,543,506$ & $0.29,095,492$ & $0.21,253,606$ & $1.22,126,511$ & $1.36,749,934$ \\
$\gamma_{1}$ (day $^{-1}$ ) & $0.99,999,988$ & $0.99,999,990$ & $0.12,575,860$ & $0.06,319,081$ & $0.99,999,967$ & $0.99,999,999$ \\
$\sigma_{1}$ (day $\left.^{-1}\right)$ & $0.15,257,834$ & $0.39,163,616$ & $0.05,079,499$ & $0.03,465,213$ & $0.15,437,778$ & $0.30,198,777$ \\
\hline
\end{tabular}



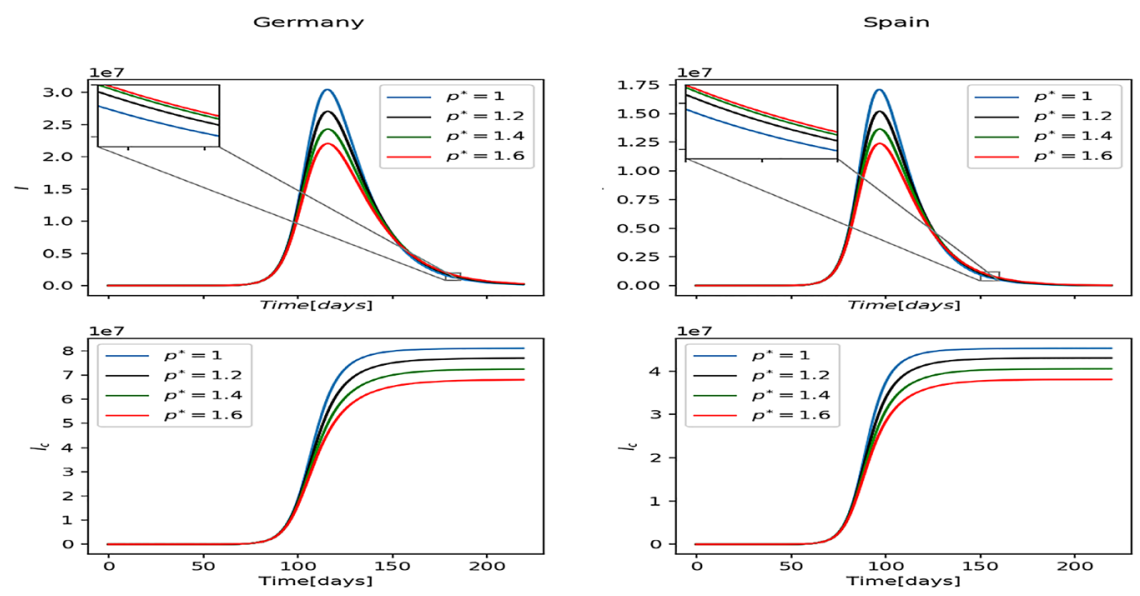

Italy
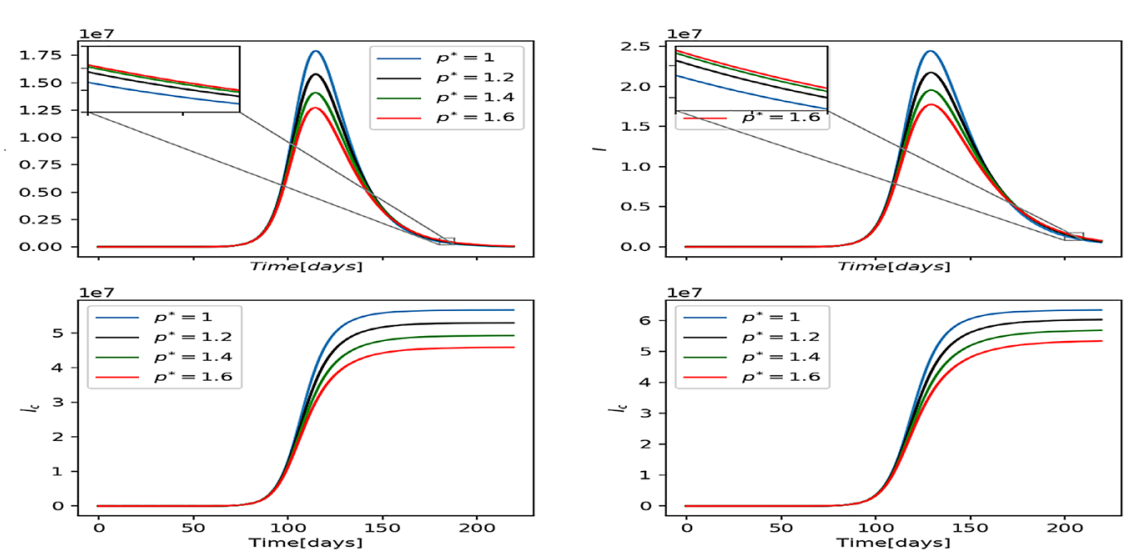

Algeria
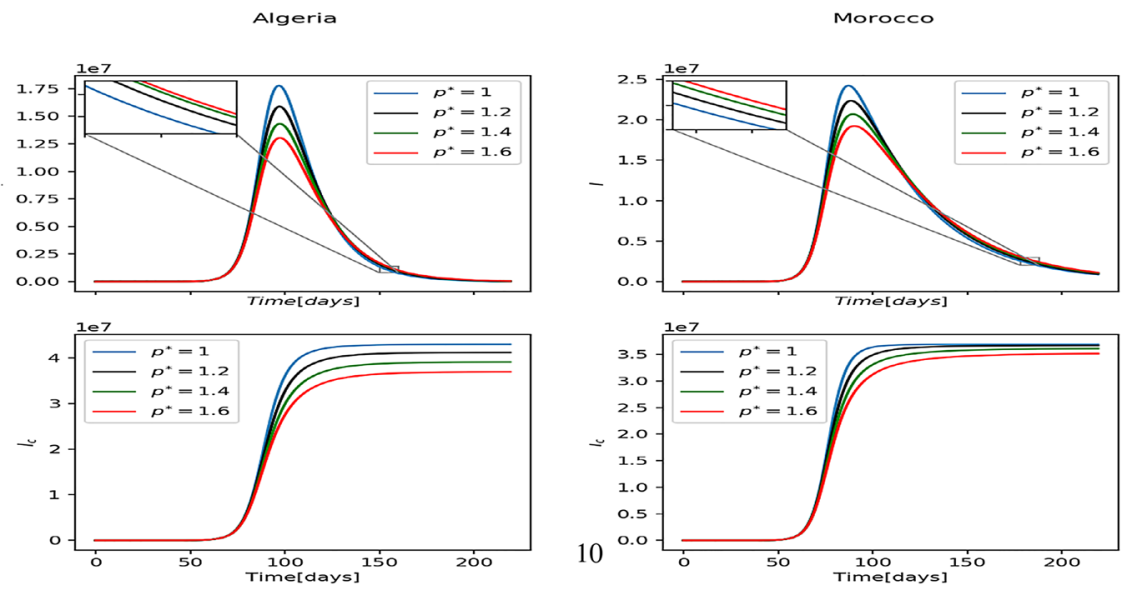

Figure 4.1: Numerical simulation of our model with the parameters $\beta, \gamma$ and $\sigma$ which are computed by fitting SIRD model during the first 30 days. The first and the third rows represent the infected cases, the second and the last rows are the cumulative cases. We investigate the effect of changing the parameter $p^{\star}$ in the simulation of COVID-19 spreading for various countries (recall that $p^{\star}=1$ corresponds to the classical SIRD model).

non-linear proposed model (3.1): reducing the contact $\mathcal{L}\left(., \frac{S}{N}\right) I$. To estimate the impacts on the non-linearity by these measures, we develop several numerical simulations of the effects of these measures by adjusting the estimates of the parameter $p^{\star}$. 


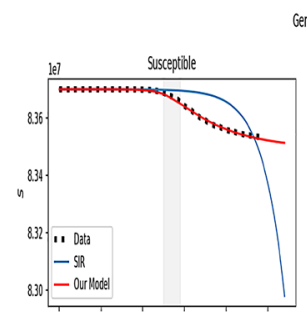

Germany
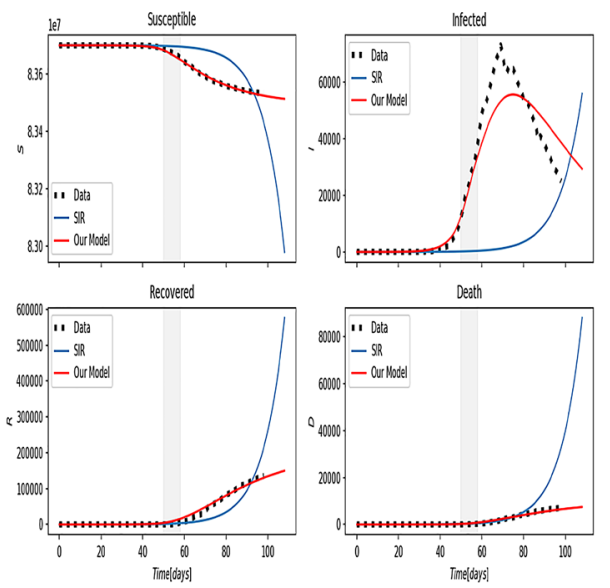

Deth
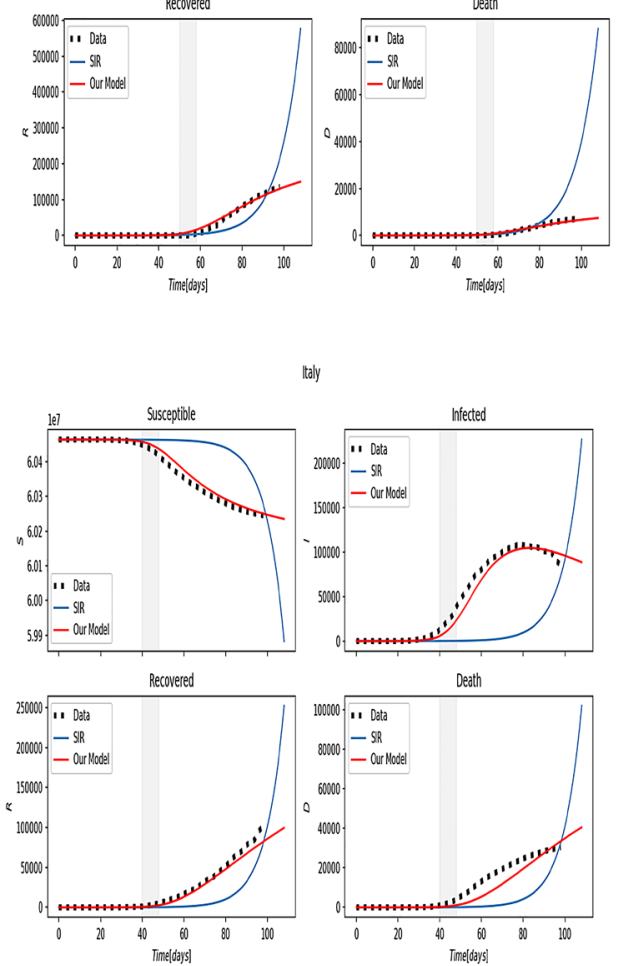

Algeria

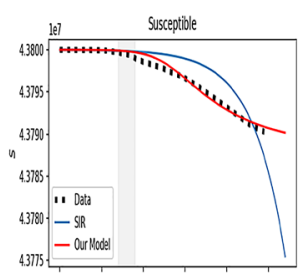

ecovered

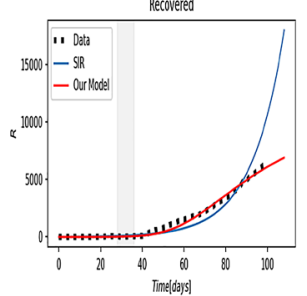

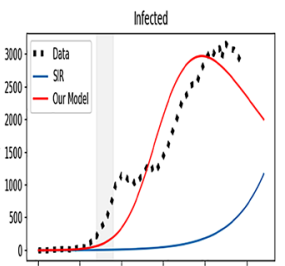

Ded

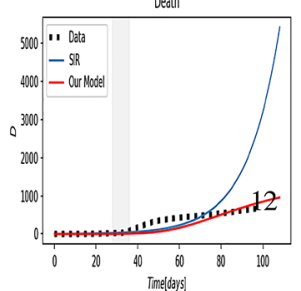

Spain

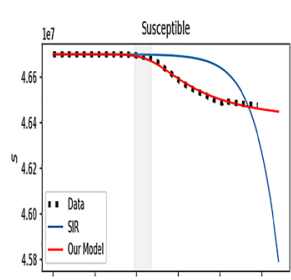

Recovered
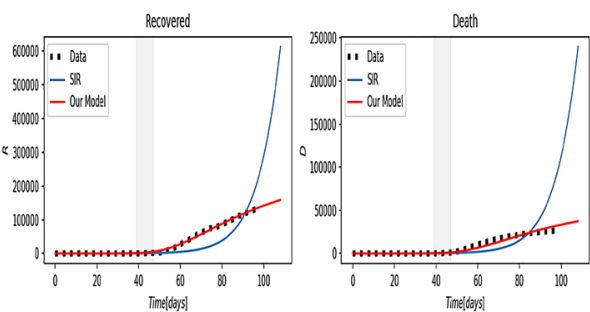

France
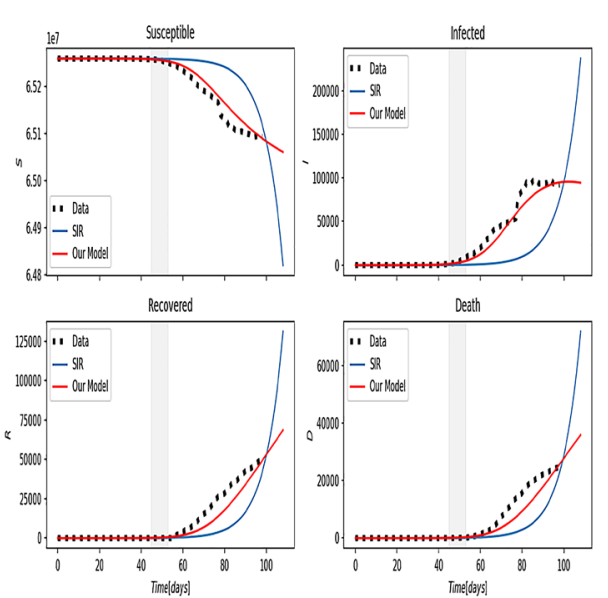

Morocco
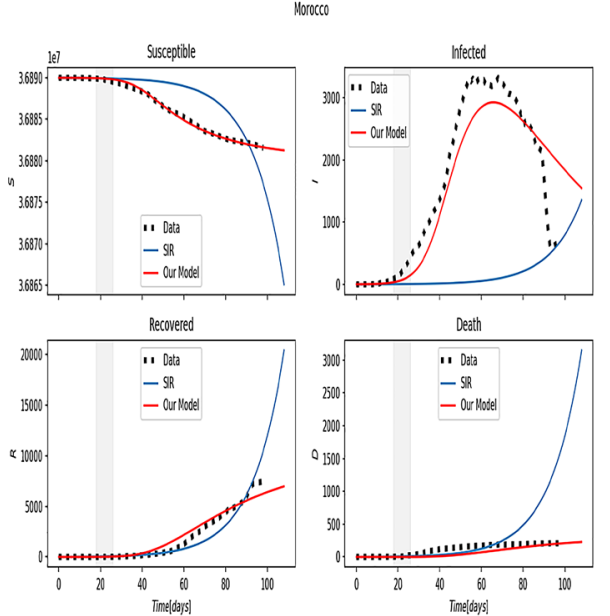

Figure 4.2: Fit of data from Germany, Spain, Italy, France, Algeria and Morocco with classical SIR and the proposed model. The black dots are the susceptible, infective, recovered and death reported cases. 


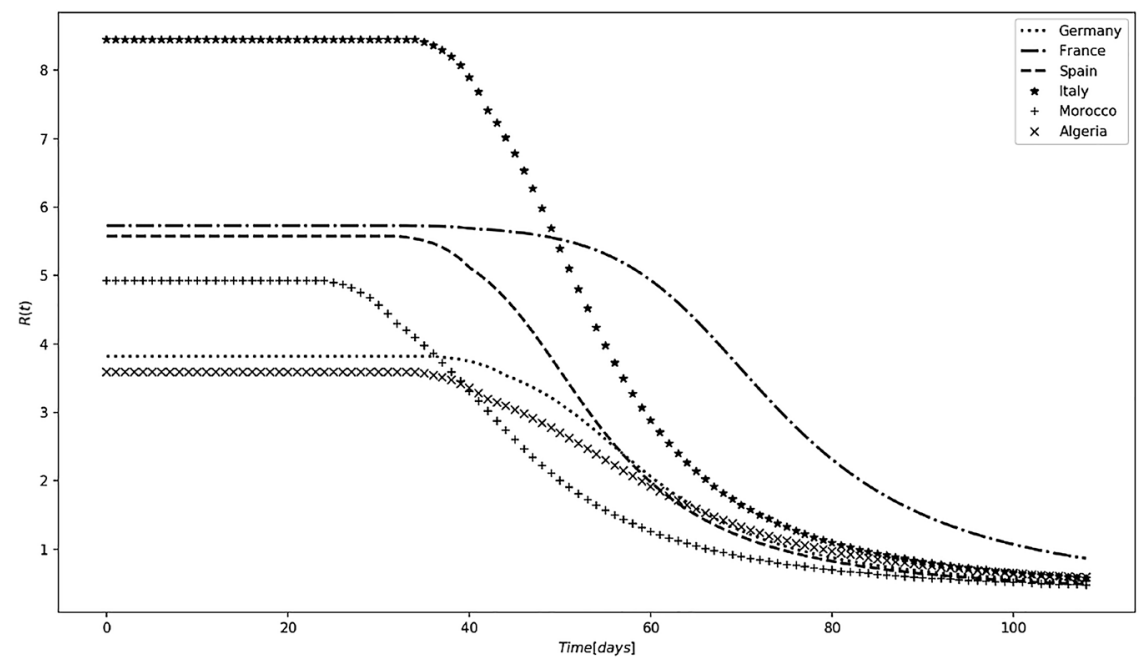

Figure 4.3: Simulation of the reproduction number $\mathcal{R}_{t}^{p}$ from (4.3) for the previous countries.

Now we illustrate various numerical tests based on some real data. We consider the problem (3.1) with

$$
\mathcal{L}(t, r)=r_{\varepsilon}^{p_{\varepsilon}^{\delta}(t)}, \quad \text { with } p_{\varepsilon}^{\delta}(t)= \begin{cases}1+\varepsilon & \text { if } t \leq t_{c}, \\ \frac{p^{\star}-1-\varepsilon}{\delta}\left(t-t_{c}\right)+1+\varepsilon & \text { if } t_{c} \leq t \leq t_{c}+\delta, \\ p^{\star} & \text { if } t \geq t_{c}+\delta .\end{cases}
$$

In the following, we assume that $\varepsilon=10^{-6}, \delta=8$ days and $t_{c}$ is the beginning time of lockdown announced by the authorities in each country. In the first test, we use our algorithm and we fit the SIRD model (2.4) with reported data in the period from the beginning of the outbreak until time $t_{c}$ at which social distancing is started, and we compute the the parameters $\beta, \gamma$ and $\sigma$. Then by using these parameters we simulate the infected and cumulative cases in our model (3.1) and we illustrate the effect social distancing by varying the parameter $p^{\star}$.

The previous tests illustrate the effect of social distancing represented by $p^{\star}$ on the number of infected population in various countries. In the absence of social distancing i.e. $p^{\star}=1$ the number of infected cases grows exponentially to reach a maximum infected number and also decreases exponentially. The case where $p^{\star}>1$ corresponds to various social distancing measures for e.g. closing schools and universities, isolation and home quarantine. We observe that when $p^{\star}$ increases, the impact of the pandemic is reduced by flattening the curve, reducing the peak incidence and overall the infected numbers (Figure 4.1).

In the next test, we fit the observed data and we compute the appropriate parameters of the proposed model. Additionally, we compare the fitting results with the classical SIRD model (see Table 2). This permits to evaluate the social distancing measures in each country. In Figure 4.2, we can see which of SIRD models is the best by fitting them to data. We observe that our model-fitting SIRD system with $p^{\star}>1$ is better compared to the classical case $p^{\star}=1$. Moreover, the adaptive nature of our model gives a considerable results. We plan to update these results regularly on our platform (http://vps730710.ovh.net:3000/) for various country.

Table 2 represents the obtained values of the parameters by fitting our model with reported data. The parameter $p^{\star}$ illustrate the degree of social distancing in each country. We notice that the value of the parameter $p^{\star}$ is more important in Morocco and in Algeria. This reflects the strict lockdown measures implemented in these countries.

Now, Thanks to (3.5), the effective reproduction number is given by

$$
\mathcal{R}_{t}^{p}=\frac{\beta}{\gamma+\sigma}\left[\frac{S(t)}{N}\right]^{p_{\varepsilon}^{\delta}(t)} .
$$


In the next figure, we present the evolution of $\mathcal{R}_{t}^{p}$ for each country.

In Figure 4.3, we observe the efficiency of the measure implemented by Moroccan and Algerian governments, that leads to control the outbreak and permit to decrease quickly the $\mathcal{R}_{t}^{p}$ reproduction number (recall these two countries started early to implement strict lockdown). Observe that the reproduction number $\mathcal{R}_{t}^{p}$ of Germany is close to the one of Morocco and Algeria. Note that Germany has tested for COVID-19 on a larger scale comparing to France, Spain and Italy. Consequently, Germany had a lower death rate comparing to these countries.

\section{Conclusion and perspectives}

The physical distancing or the social distancing measures have an important effects to reduce the spread of COVID-19 by limiting face-to-face contact with others. The aim of social distancing is to reduce the impact of the pandemic by flattening the curve, reducing Figure 4.1. Since the goal of governmental measures is to decrease the infection, the distancing measures need to remain in place for long period of time in the pandemic phase. In the case where little herd immunity is required, a second wave of infection could occur when the governmental interventions are lifted. In this paper, we have led a new mathematical and numerical analyses for COVID-19. This work provides a theoretical framework for public health interventions. The proposed model is an adaptive variant of the SIRD models. This model describes the spreading of coronavirus under the effect of a social distancing. Moreover, our model is more robust for fitting and for predicting the future trend of the COVID-19 outbreak. It permits to track the main model properties. The model can also be used to evaluate the the efficiency of social distancing measures and estimate the reduce the number of infected individuals. The proposed model present some classical limitations and they does not take into account the spatial distribution of each individuals and their displacement. In this case, one can extend our proposed model (3.1) to a new non homogenous model with an explicit space dependance. The simplest generalization of our model can be given by the following system:

$$
\left\{\begin{array}{l}
\frac{\partial S(t, x)}{\partial t}-d_{1} \Delta S(t, x)=-\beta\left[\frac{S(t, x)}{N}\right]^{p(t, x)} I(t, x), \\
\frac{\partial I(t, x)}{\partial t}-d_{2} \Delta I(t, x)=\beta\left[\frac{S(t, x)}{N}\right]^{p(t, x)} I(t, x)-(\gamma+\sigma) I(t, x),
\end{array}\right.
$$

where the functions $S(t, x)$ and $I(t, x)$ represents the densities and the new diffusion term $d_{i} \Delta$ describes a random motion of the individuals. Herein, the social distancing function $p(t, x)$ depend on time and space and the non homogenous model describe the dynamics well. This situation can be observed when the distancing measures has been applied differently with respect to various regions. However, this generalized model is much more complicated problem and cannot be solved directly by our algorithm, for that in the future work we intend to analyse mathematically this model and also solve numerically this problem by using our algorithm combined with the BDF scheme developed recently in (Bouchriti et al. 2020).

Research funding: None declared.

Author contribution: All authors have accepted responsibility for the entire content of this manuscript and approved its submission.

Competing interests: Authors state no conflict of interest.

Informed consent: Informed consent was obtained from all individuals included in this study.

Ethical approval: The local Institutional Review Board deemed the study exempt from review.

\section{References}

Anderson, R. M. 1991. “Discussion: The Kermack-McKendrick Epidemic Threshold Theorem.” Bulletin of Mathematical Biology 53 (1): 3-32. 
Bouchriti, A., M. Pierre, and N. Alaa. 2020. “Gradient Stability of High-Order BDF Methods and Some Applications.” Journal of Difference Equations and Applications 26 (1): 74-103.

Bertozzi, A. L., E. Franco, G. Mohler, M. B. Short, and D. Sledge. 2020. "The Challenges of Modeling and Forecasting the Spread of COVID-19.” Proceedings of the National Academy of Sciences 117 (29): 16732-8.

Bootsma, M. C. J., and N. M. Ferguson. 2007. "The Effect of Public Health Measures on the 1918 Influenza Pandemic in U.S. Cities.” Proceedings of the National Academy of Sciences 104 (18): 7588-93.

Chou, C.-S., and A. Friedman. 2016. Introduction to Mathematical Biology. Modeling, Analysis, and Simulations. Springer Undergraduate Texts in Mathematics and Technology, vii+172. Cham: Springer.

Chowell, G. 2017. "Fitting Dynamic Models to Epidemic Outbreaks with Quantified Uncertainty: A Primer for Parameter Uncertainty, Identifiability, and Forecasts.” Infectious Disease Modelling 2: 379-98.

Driessche, P., and J. Watmough. 2002. "Reproduction Numbers and Sub-threshold Endemic Equilibria for Compartmental Models of Disease Transmission." Mathematical Biosciences 180: 29-48.

Eksina, C., K. Paarpornb, and J. S. Weitz. 2019. "Systematic Biases in Disease Forecasting - The Role of Behavior Change.” Epidemics 27: 96-105.

Ferguson, N. M., D. Laydon, G. Nedjati-Gilani, N. Imai, K. Ainslie, M. Baguelin, S. Bhatia, A. Boonyasiri, Z. Cucunubá, G. Cuomo-Dannenburg, and A. Dighe. 2020. Impact of Non-Pharmaceutical Interventions (NPIs) to Reduce COVID-19 Mortality and Healthcare Demand. London: Imperial College COVID-19 Response Team, Report 9, March, 16.

Friedman, A. 2018. Mathematical Biology. Modeling and Analysis. CBMS Regional Conference Series in Mathematics, Vol. 127, viii+100. Washington, DC, Providence, RI: Conference Board of the Mathematical Sciences, American Mathematical Society.

Hethcote, H. W. 2000. “The Mathematics of Infectious Diseases.” SIAM Review 42 (4): 599-653.

Kermack, W. O., and A. G. McKendrick. 1927. "A Contribution to the Mathematical Theory of Epidemics.” Proceedings of the Royal Society 115 (772): 700-21.

Liu, X., and P. Stechlinski. 2017. Infectious Disease Modeling: A Hybrid System Approach Nonlinear Systems and Complexity Book 19. Cham: Springer International Publishing, Springer.

Murray, J. D. 2002. Mathematical Biology. I. An Introduction, Interdisciplinary Applied Mathematics, vol 17, 3rd ed. New York: Springer-Verlag.

Weiss, H. 2009. A Mathematical Introduction to Population Dynamics. Rio de Janeiro: Instituto Nacional de Matemática Pura e Aplicada (IMPA). 